\title{
Fast atrial fibrillation and caecal volvulus - a case report and evidence based management
}

\author{
Alexander Liu, ${ }^{1}$ Edward Nicol² \\ 1Department of Gastroenterology, Chelsea and Westminster Hospital, London, UK; \\ 2Department of Cardiology, Royal Brompton Hospital, London, UK
}

Correspondence to Dr Alexander Liu, alexander.liu104@ic.ac.uk

\section{Summary}

An 81-year-old Caucasian lady with permanent atrial fibrillation (AF) was admitted with palpitations and fast AF. She took bisoprolol and warfarin with subtherapeutic international normalised ratio. Rate control of AF was successful. Around $36 \mathrm{~h}$ later, she developed abdominal pain and vomiting. A caecal volvulus was diagnosed on CT. She underwent emergency laparotomy. Intraoperatively, an obstructing tumour was found in the colonic hepatic flexure. It was deemed inoperable. The caecal volvulus was decompressed and an ileo-transverse colon bypass was performed. She made a good recovery and her fast AF settled postoperatively. She was seen by the oncology team and was discharged with palliative care support with no further exacerbation of AF.

\section{BACKGROUND}

\section{Atrial fibrillation - an age old disease}

Atrial fibrillation (AF) and atrial flutter (AFL) are the commonest narrow complex arrhythmias. They are known to complicate underlying malignancies. ${ }^{1}$ Liu and Pusalkar described a case of new onset AFL in a patient with glioblastoma multiforme. However, the authors concluded that the association was likely to be coincidental. AF is the most prevalent arrhythmia which affects $1-2 \%$ of the population. $^{2}$ It is age dependent, affecting less than $0.5 \%$ of people in their fourth decade, rising to more than $10 \%$ in subjects over 80 years old. ${ }^{2}$ Despite extensive research into its pathogenesis and management, the incidence and prevalence of AF is increasing. ${ }^{3}$ According to both the National Institute of Clinical Excellence guidelines (NICE) and the 2006 American Heart Association/American College of Cardiology/European Society of Cardiology guidelines (AHA/ACC/ESC); AF is classified into three groups. ${ }^{3}$ Paroxysmal AF lasts less than 7 days and terminates spontaneously while persistent AF lasts more than 7 days and may terminate spontaneously or are successfully cardioverted to normal sinus rhythm (NSR). ${ }^{3}$ Permanent $\mathrm{AF}$ is present if the arrhythmia lasts more than 7 days and cannot be cardioverted back into NSR. ${ }^{3}$ However, these terms are not mutually exclusive. The duration of AF reflects the length of each individual episode as well as the total amount of time a patient has suffered from AF since diagnosis. ${ }^{3}$ For this reason, a patient who suffers many years from recurrent episodes of AF with spontaneously terminations can be classified as paroxysmal. ${ }^{3}$

The pathogenesis of AF is complex. Initiation is likely due to a combination of atrial anatomical pathology as well as electromechanical remodelling. ${ }^{3}$ Atrial fibrosis and atrial muscle loss are present in early AF and can be demonstrated in biopsies. Atrial fibrosis occurs adjacent to normal conducting atrial tissue, providing heterogeneous conduction substrates for arrhythmogenesis. ${ }^{4}$ Patients with severe atrial fibrosis are less likely to respond to cardioversion than those with mild to moderate fibrosis. ${ }^{3}$ Atrial dilatation is also implicated in the onset and perpetuation of AF, which may be caused by activation of the renin-angiotensinaldosterone system (RAAS). ${ }^{3}$ Propagation of AF worsens any atrial dilatation present at onset, leading to decreased contractility and reduced atrial compliance. ${ }^{3}$ Interestingly, atrial stretch induced by AF upregulates RAAS and transforming growth factor $\beta-1$, contributing to further atrial fibrosis. ${ }^{5}$ These structural changes are likely to provide the substrate for electrical disturbances.

There are two hypothesised mechanisms for the electrical disturbances in AF: the automatic focus theory and the multiple wavelet theory. ${ }^{3}$ In the former, there is a single arrhythmogenic focus or multiple foci within a discreet area. ${ }^{3}$ The commonest region is near the junction between the left atrium and the pulmonary vein (LA-PV), owing to the shorter refractory period of atrial tissue in the PV. ${ }^{6}$ Indeed patients with AF have shorter refractory periods within their PV compared to controls. ${ }^{6}$ Rapid-firing from foci within the PV may lead to micro-reentry, exacerbated by shorter refractory periods. ${ }^{7}$ Catheter ablation near the LA-PV junction has led to successful termination of AF in selected patients. ${ }^{3}$

The multiple wavelet theory, first described by Moe and colleague, refers to chaotic firing of multiple wavefronts within the atria from several foci. ${ }^{8}$ The number of wavefronts is increased by a large atrial mass, reduced conduction velocities and shortened refractory periods. ${ }^{3}$ This theory explains why atrial fibrosis (reduced conduction velocity) and atrial dilatation (large atrial mass) predispose to $\mathrm{AF}^{3}$ There also appears to be a gradual, albeit controversial, progression from paroxysmal to persistent AF culminating finally to permanent $\mathrm{AF}^{3}$

Caecal volvulus - a rare surgical phenomenon in practice? Caecal volvulus is defined as torsion of the caecum on its mesentery, leading to nearly $1 \%$ of all cases of bowel obstruction. ${ }^{9}$ Causes include abnormal colonic fixation 


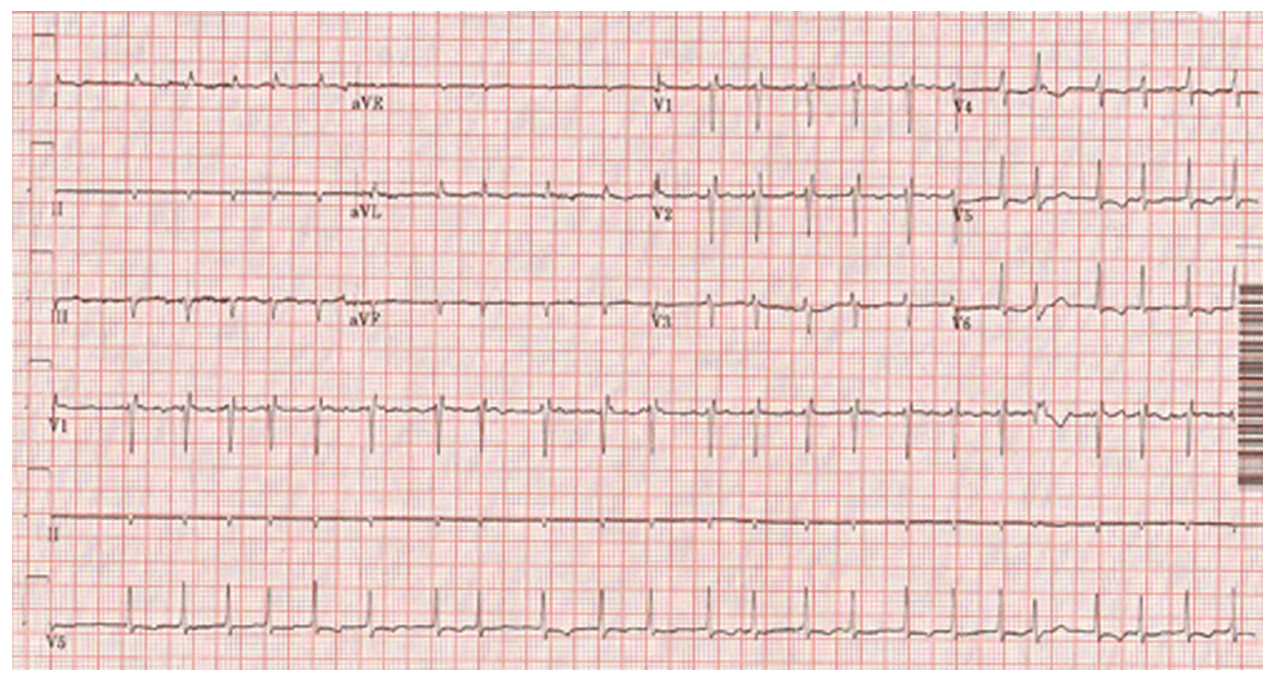

Figure 1 A 12-lead ECG taken on admission showing fast atrial fibrillation with a ventricular rate of approximately 138/min.

to the peritoneum to allow a free segment to twist on itself. ${ }^{9}$ In addition, abdominal masses and adhesions can serve as rotatory fulcrums. ${ }^{9}$ The most serious complication is closed-loop obstruction leading to bowel necrosis and death if prompt surgical management is delayed. ${ }^{10}$ Presentations are usually those of bowel obstruction: vomiting, constipation, colicky abdominal pain and distension. ${ }^{11}$ Classically, caecal volvulus can be diagnosed on abdominal radiographs as isolated distended bowel loop resembling a large 'coffee bean'. ${ }^{12}$ This loop usually localises to the left side. ${ }^{12}$ However, this is unpredictable and a CT is required to distinguish between caecal and sigmoid volvulus. ${ }^{9}$ The definitive management is surgical intervention with laparotomy and detorsion of the volvulus. ${ }^{9}$ The usual practice is detorsion with an ileocolic resection with ileocolic anastomosis, with no chance of recurrence. ${ }^{9}$

\section{CASE PRESENTATION}

An 81-year-old Caucasian lady with a 30 years history of permanent AF, type II diabetes mellitus and diverticular disease presented at 3 a.m with sudden onset palpitations which woke her up from sleep. She also had shortness of breath. There was no chest pain, cough, sputum or haemoptysis. She had no urinary symptoms and there was no change in bowel habit. She took warfarin for AF, bisoprolol, metformin. Her diverticular disease was inactive for 7 years. There was no history of ischaemic heart disease, hypertension or cerebrovascular disease. She was a nonsmoker with an unremarkable family history. On initial assessment, she was alert and orientated with a tachycardia at $138 / \mathrm{min}$, auricular temperature of $36.6^{\circ} \mathrm{C}$, blood pressure $95 / 42 \mathrm{~mm} \mathrm{Hg}$, respiratory rate $22 / \mathrm{min}$ and pulse oximetry was $96 \%$ on room air. On examination, her pulse was irregularly irregular. Jugular venous pulse was $2 \mathrm{~cm}$. Normal first and second heart sounds were present with no murmurs or added sounds on auscultation. Diffused fine inspiratory crepitations were heard bilaterally throughout the chest. On admission, her abdomen was soft and nontender and she was neurologically intact.

Her initial bloods were as follows: haemoglobin $14 \mathrm{~g} / \mathrm{dl}$, white cell count $6.5 \times 10^{9} / 1$, platelet $249 \times 10^{9}$, C-reactiveprotein $12.7 \mathrm{mg} / 1$, sodium $141 \mathrm{mmol} / 1$, potassium $4.4 \mathrm{mmol} / 1$, urea $7 \mathrm{mmol} / \mathrm{l}$, creatinine $62 \mathrm{mmol} / \mathrm{l}$, corrected calcium $2.47 \mathrm{mmol} / \mathrm{l}$, international normalised ratio (INR) 0.9 (target range 2-3). Her liver function and thyroid function tests were normal. Serum troponin I was $<0.032 \mu \mathrm{g} / 1$ on admission and again at $12 \mathrm{~h}$ after the onset of palpitations. Arterial blood gas sample showed metabolic acidosis, hypoxia and raised lactate. She was given oxygen therapy. Her ECG showed fast AF with ventricular rate of $138 / \mathrm{min}$ (figure 1). A urine dipstick was unremarkable and her chest radiograph showed evidence of pulmonary fibrosis with cardiomegaly despite antero-posterior film (figure 2).

She was monitored on telemetry. AF strict rate control was attempted with bisoprolol and digoxin which reduced her ventricular rate to between 80 and 110 for the next 48 h. Her subtherapeutic INR was noted and she was given increased doses of warfarin with low-molecular weight heparin cover until therapeutic. A transthoracic echocardiogram showed a right atrial size of $5.5 \mathrm{~cm}$ with left ventricular ejection fraction of $50 \%$. There was evidence of trivial mitral regurgitation.

Thirty-six hours after admission, the patient developed sudden onset right iliac fossa pain $3 \mathrm{~h}$ after a light lunch with faecolant vomiting. On examination, her abdomen was distended with guarding and tenderness over the right iliac fossa. No rebound tenderness or masses were detected. Bowel sounds were hyperactive. Digital rectal examination was unremarkable. Abdominal radiograph showed right-sided large bowel distension indicative of caecal volvulus (figure 3), which was confirmed with CT abdomen/pelvis (figure 4). The CT scan also showed ischaemic changes surrounding the volvulus and the large bowel wall. She was reviewed by the surgical team and underwent an emergency laparotomy. Intraoperatively, an obstructing tumour was found in the hepatic flexure inseparable from the duodenum and pancreas. It was deemed inoperable. Her caecal volvulus was decompressed via an appendicectomy. An ileo-transverse colon bypass was carried out.

\section{OUTCOME AND FOLLOW-UP}

Postoperatively, the patient made a good recovery in the intensive care unit (ITU). On the second day in ITU, 


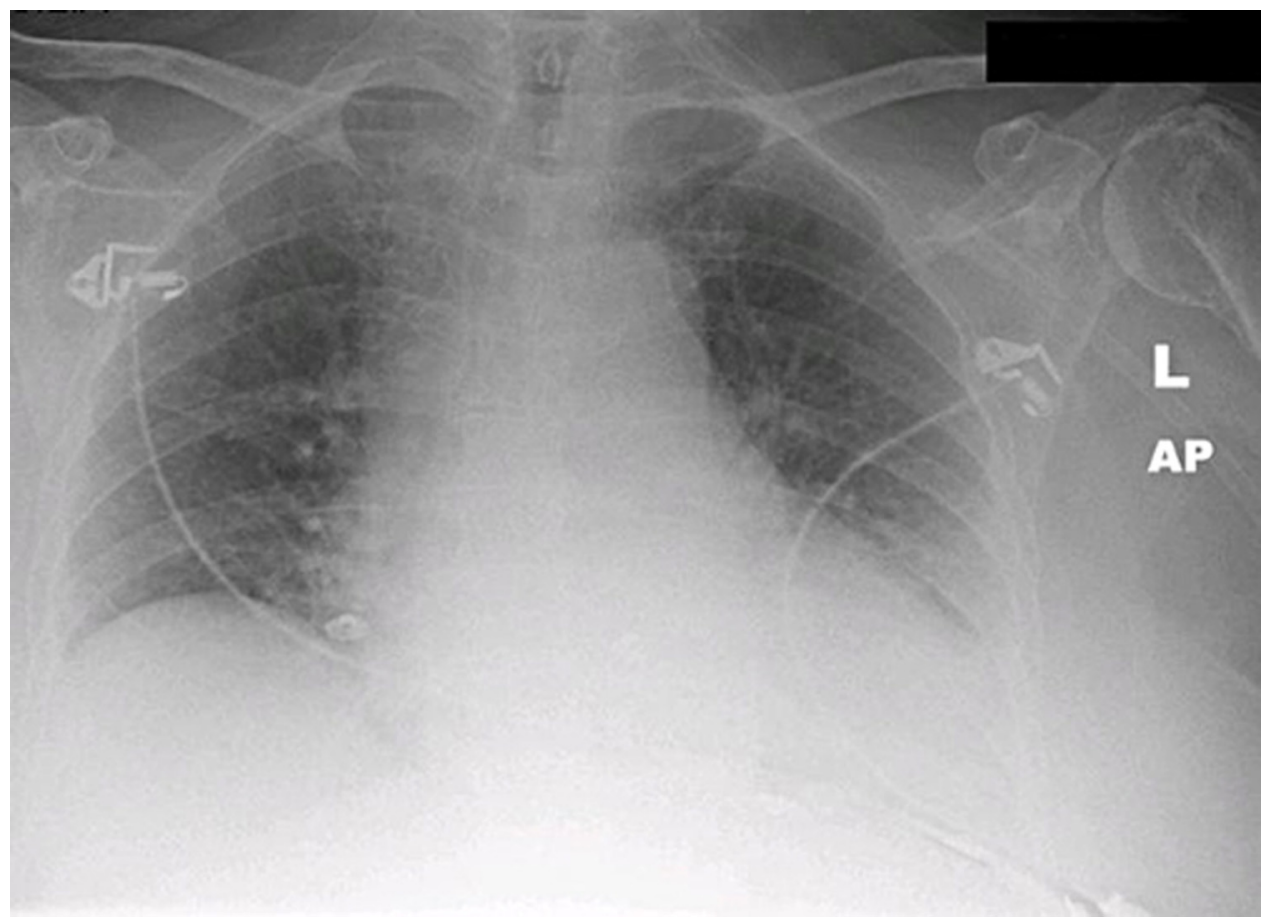

Figure 2 Chest radiograph on admission showing cardiomegaly and fibrotic changes.

her fast AF was well controlled with normal ventricular response below $80 / \mathrm{min}$ on digoxin and bisoprolol, which were continued. She was referred to the oncology team for the management of malignancy, which unfortunately never obtained a histological diagnosis since no biopsies were taken intraoperatively. She was later found to have profoundly raised CA19.9 and carcinoembryonic antigen tumour markers. A lengthy discussion took place between the oncology consultant, the patient and her relatives and a joint decision was made for palliative management given her age and co-morbidities. She was discharge a week later with palliative care services and follow-up with the oncology team. She did not have another relapse of fast AF.

\section{DISCUSSION}

We described an older lady who presented with fast AF and subsequently developed caecal volvulus most likely secondary to an obstructing tumour in the hepatic flexure. This case demonstrates two interesting principles. First, it gives an account of the challenging task of managing fast $\mathrm{AF}$ in older patients. The successful management of AF in the acute setting is sometimes difficult and an evidence based outline is not always available. Second, this case demonstrates the unpredictable nature of clinical practice where two rather contrasting ailments (AF and caecal volvulus) can coexist. This necessitates an open minded approach in cardiology; a reminder of an important clinical lesson.

\section{The NICE management of AF}

AF management can be broadly divided into rate or rhythm control. The latter aims to restore NSR. Stroke prevention should be carried out with either intervention. ${ }^{3}$ According to the NICE guidelines, provided that adequate thromboprophylactic measures are taken, permanent AF should be managed with rate control and paroxysmal AF with rhythm control. Persistent AF can be managed with either rate or rhythm control depending on patient related factors. Rhythm control is preferred in younger, patients with symptoms with first presentation of lone AF or those with congestive heart failure. Rhythm control should also be attempted if AF is secondary to treated precipitants. Rate control is better indicated in sufferers of persistent AF if the patient is older (over 65 years), with coronary artery disease and unsuitable for cardioversion and antiarrhythmic drugs.

\section{Rate control versus rhythm control}

Two landmark non-inferiority trials AFFIRM $^{13}$ (Atrial Fibrillation Follow-up Investigation of Rhythm Control) and $\mathrm{RACE}^{14}$ (Rate Control versus Electrical Cardioversion for Persistent Atrial Fibrillation) have helped to solve the age old conundrum of rate versus rhythm control. The AFFIRM trial enrolled 4060 patients randomised to receive either rate control or rhythm control. The intervention in the latter arm of the study was achieved with antiarrythmic drugs and direct-current cardioversion. ${ }^{13}$ Stroke prevention with warfarin (target INR 2-3) was offered to all rate controlled subjects and those in the rhythm control group with a minimum period of 4-12 weeks in NSR. ${ }^{13}$ There was no significant difference in the 5 -year mortality between the rate controlled and rhythm controlled arms; $21.3 \%$ versus $23.8 \%$, respectively. CI of 0.99 to 1.34 ( $p=0.08) .{ }^{14}$ The stroke rates were also comparable among the rate controlled and rhythm controlled patients. ${ }^{13}$ The RACE trial was a randomised controlled trial of 522 patients comparing rate control and rhythm control. The authors concluded that $\mathrm{AF}$ rate control was not inferior to rhythm control in reaching primary end points. ${ }^{14}$ These end points included cardiovascular death, heart failure hospitalisations, severe bleeding, thromboembolism, pacemaker insertion and 


\section{BMJ Case Reports}

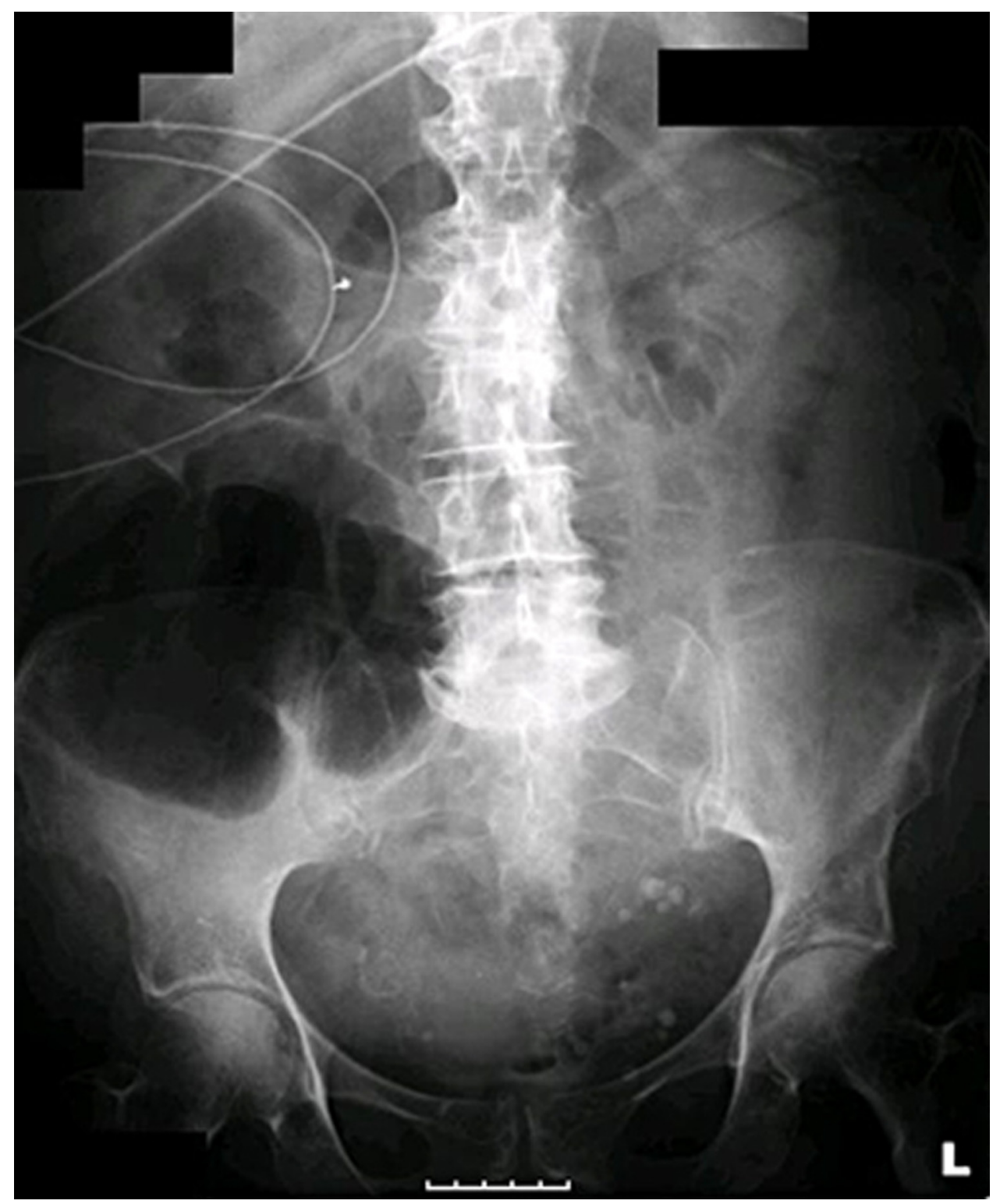




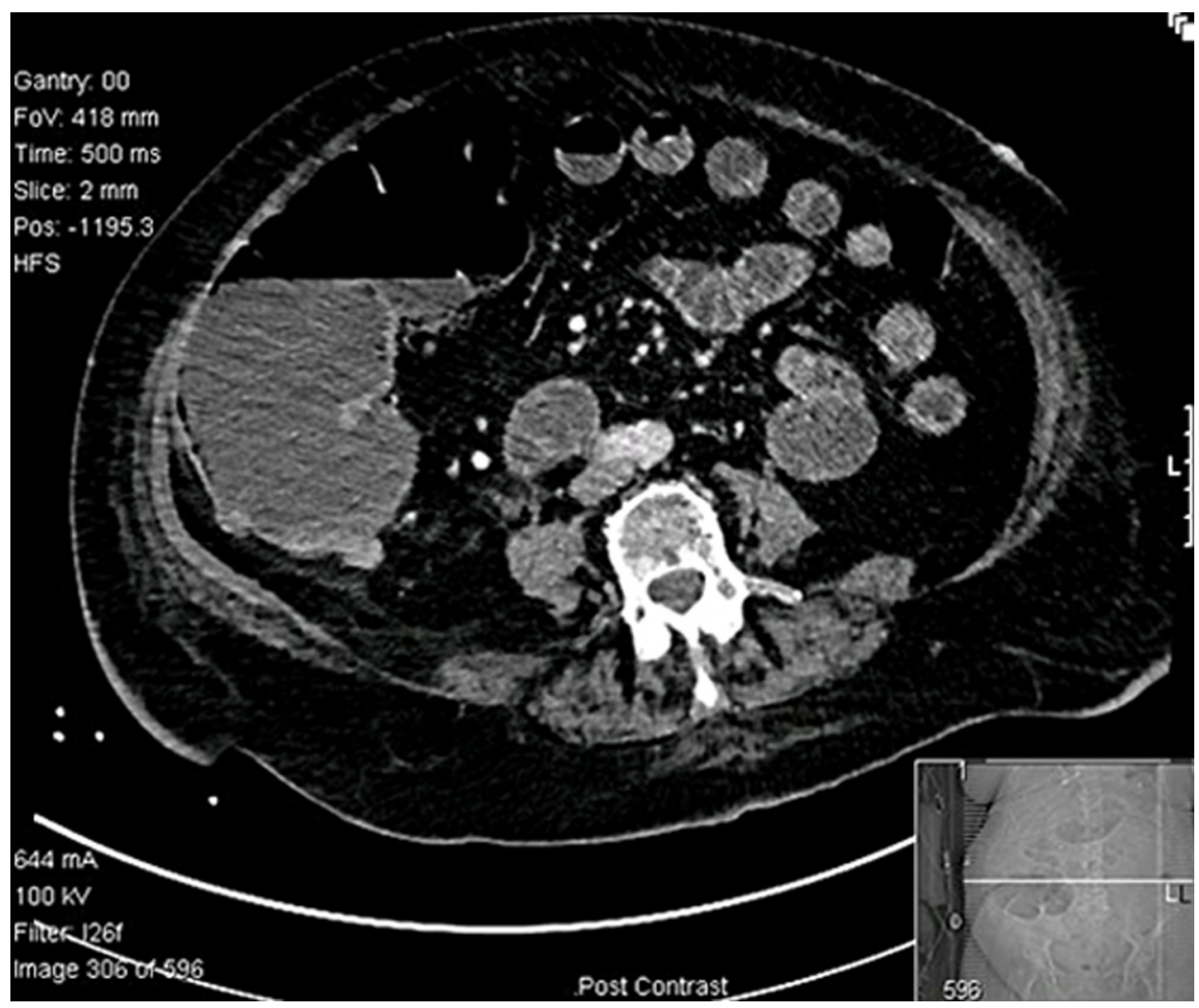

Figure 4 CT demonstrating a caecal volvulus.

We were partially successful in the first $48 \mathrm{~h}$ with the use of $\beta$-blocker (bisoprolol) and digitalis; the rate was maintained between 80 and 110/min. Our intentions of strict rate control contradicted with the ESC guidelines recommendations. ${ }^{15}$ However, the results of our interventions was within the limits of lenient rate control (<110/min). It was not appropriate to adopt rhythm control in permanent AF which is unlikely to cardiovert to NSR.

\section{Stroke prevention in AF and the future}

Stroke prevention is essential in AF and $>95 \%$ originate from the left atrial appendage. Risk stratification can be achieved with the $\mathrm{CHADS}_{2}$ (congestive heart failure, hypertension, age over 75 , diabetes and stroke) score, where a history of stroke or transient ischaemic attack allocates 2 points. ${ }^{15} 17$ The ACCF/AHA/HRS guidelines recommend the use of aspirin if $\mathrm{CHADS}_{2}$ score is 0 , aspirin or warfarin if score is 1 , warfarin if score is 2 or above. ${ }^{17}$ The target INR is 2.5 (range 2.0 to 3.0 ). ${ }^{18} 19$ Our patient scored two points (age and diabetes) on CHADS2 and was taking long-term warfarin. However, her INR was subtherapeutic on admission, which required supplementation with LMWH.

Maintaining warfarin within its therapeutic range is sometimes difficult due to the abundant numbers of interactions with other drugs and poly-pharmacy in the older population. This calls for alternative drug for stroke prevention in AF. The extensive evaluation of evidence is beyond the scope of this case report; we will outline the evidence for dabigatran (oral direct thrombin inhibitor) and rivaroxaban (oral factor Xa inhibitor).

The randomised evaluation of long-term anticoagulation therapy (RE-LY) trial enrolled 18113 patients randomised to either warfarin (INR 2.0-3.0) or twice daily 110 or 150 mg dabigatran with a 2 year follow-up period. ${ }^{19}$ This was a non-inferiority trial using stroke or systemic emboli as primary end points. There was no significant difference in the number of strokes between the warfarin and dabigatran treated arms. ${ }^{19}$ Haemorrhagic stroke rate was lower in the dabigatran treated arm. ${ }^{19}$ The risk of myocardial infarction (MI) was reported to be higher in the dabigatran treated group, however, introduction of revised data failed to show an increased risk of MI in patients treated with $150 \mathrm{mg}$ of dabigatran compared to placebo. ${ }^{20}$ The investigators concluded that dabigatran may be a suitable alternative to warfarin therapy, with a rapid onset of action $(2 \mathrm{~h})$ and no need for blood test monitoring. ${ }^{19}$ ACCF/AHA/HRS concurred with this recommendation and dabigatran was licensed by the Food and Drug Administration (FDA). ${ }^{17}$ Further cost-effective analysis is needed and we await further updates on the NICE guidelines.

The Rivaroxaban Once-daily Oral Direct Factor Xa Inhibition Compared with Vitamin $\mathrm{K}$ antagonism for the Prevention of Stroke and Embolism Trial in Atrial Fibrillation (ROCKET-AF) trial showed that rivaroxaban was not inferior to warfarin for AF thromboprophylaxis. ${ }^{21}$ Bleeding complications were comparable between the two treatment groups. ${ }^{21}$ The complete results have yet been published. Rivaroxaban is licensed for venous thromboprophylaxis in patients undergoing hip and knee replacements ${ }^{21}$ but is not yet approved for the use in AF, although an application has been made for the FDA in January 2011.

Other drugs have been considered but not recommended as alternatives to warfarin in $\mathrm{AF}$ due to adverse effects or lack of efficacy, these include clopidogrel (ineffective 
stroke prevention) ${ }^{22}$ and ximelagatran (angina and coronary ischaemia). ${ }^{23}$

\section{CONCLUSION}

\section{AF and caecal volvulus - feasible connections?}

There are no cases connecting AF to caecal volvulus in the literature. $\mathrm{AF}$ is linked to mesenteric ischaemia and the pathophysiology is thromboembolic. ${ }^{24}$ Mesenteric ischaemia can result from volulus but it is unlikely to cause caecal volvulus. The CT scan of our patient demonstrated large bowel wall ischaemia. This was probably due to both the caecal volvulus and emboli from AF. The latter was supported by the subtherapeutic INR level at presentation and to some extent the aggressive attempt at rate control in the first $24 \mathrm{~h}$. The management of our patient was palliative since the tumour was locally invasive. Interestingly, her fast AF did not relapse after decompression of the volvulus, suggesting the hint of a tentative causal link. AF is known to be worsened by metabolic acidosis according to some early work in dogs; definitive data in human is limited. ${ }^{22}$ Overall, the connection between AF and caecal volvulus is controversial and hypothetical.

\section{Learning points}

Permanent AF is sometimes difficult to manage and patients can present with acute deterioration.

- Rate control is preferred in the management of permanent $A F$ and lenient rate control is not inferior to strict rate control.

- Stroke prevention is important in AF management and high risk patients are managed with warfarin, although alternative drugs have been under investigation and may be indicated in the future.

- Caecal volvulus is relatively rare and is associated with large bowel tumours.

- The presence of AF and caecal volvulus in this case illustrates the need for clinicians to keep an open mind when managing medical problems in the acute medical setting.

\section{Competing interests None.}

Patient consent Obtained.

\section{REFERENCES}

1. Liu A, Pusalkar P. Atrial flutter in a patient with glioblastoma multiforme: problems in treating thromboembolic complications. BMJ Case Reports 2011:10.1136/bcr.03.2011.3931.

2. Stewart S, Hart CL, Hole DJ, et al. Population prevalence, incidence, and predictors of atrial fibrillation in the Renfrew/Paisley study. Heart 2001;86:516-21.

3. Fuster V, Rydén LE, Cannom DS, et al. ACC/AHA/ESC 2006 guidelines for the management of patients with atrial fibrillation-executive summary: a report of the American College of Cardiology/American Heart Association Task Force on Practice Guidelines and the European Society of Cardiology Committee for Practice Guidelines (Writing Committee to Revise the 2001 Guidelines for the Management of Patients With Atrial Fibrillation). J Am Coll Cardiol 2006:48:854-906.

4. Frustaci A, Chimenti C, Bellocci F, et al. Histological substrate of atrial biopsies in patients with lone atrial fibrillation. Circulation 1997:96:1180-4.

5. Pokharel S, van Geel PP, Sharma UC, et al. Increased myocardial collagen content in transgenic rats overexpressing cardiac angiotensin-converting enzyme is related to enhanced breakdown of $\mathrm{N}$-acetyl-Ser-Asp-Lys-Pro and increased phosphorylation of Smad2/3. Circulation 2004;110:3129-35.

6. Jaïs $\mathbf{P}$, Hocini M, Macle L, et al. Distinctive electrophysiological properties of pulmonary veins in patients with atrial fibrillation. Circulation 2002;106:2479-85

7. Takahashi Y, lesaka Y, Takahashi A, et al. Reentrant tachycardia in pulmonary veins of patients with paroxysmal atrial fibrillation. J Cardiovasc Electrophysiol 2003;14:927-32.

8. Moe GK, Abildskov JA. Atrial fibrillation as a self-sustaining arrhythmia independent of focal discharge. Am Heart J 1959;58:59-70.

9. Habre J, Sautot-Vial N, Marcotte C, et al. Caecal volvulus. Am J Surg 2008:196:e48-9.

10. Ballantyne GH, Brandner MD, Beart RW Jr, et al. Volvulus of the colon. Incidence and mortality. Ann Surg 1985;202:83-92.

11. Madiba TE, Thomson SR. The management of cecal volvulus. Dis Colon Rectum 2002;45:264-7.

12. Anderson JR, Mills JO. Caecal volvulus: a frequently missed diagnosis? Clin Radiol 1984;35:65-9.

13. Wyse DG, Waldo AL, DiMarco JP, et al.; Atrial Fibrillation Follow-up Investigation of Rhythm Management (AFFIRM) Investigators. A comparison of rate control and rhythm control in patients with atrial fibrillation. N Engl J Med 2002;347:1825-33.

14. Van Gelder IC, Hagens VE, Bosker HA, et al. A comparison of rate control and rhythm control in patients with recurrent persistent atrial fibrillation. $N$ Engl J Med 2002;347:1834-40.

15. Camm AJ, Kirchhof P, Lip GY, et al.; European Heart Rhythm Association; European Association for Cardio-Thoracic Surgery. Guidelines for the management of atrial fibrillation: the Task Force for the Management of Atrial Fibrillation of the European Society of Cardiology (ESC). Eur Heart J 2010;31:2369-429.

16. Van Gelder IC, Groenveld HF, Crijns HJ, et al. Lenient versus strict rate control in patients with atrial fibrillation. N Eng/ J Med 2010;362:1363-73.

17. Wann LS, Curtis AB, Ellenbogen KA, et al.; American College of Cardiology Foundation/American Heart Association Task Force. 2011 ACCF/AHA/HRS focused update on the management of patients with atrial fibrillation (update on Dabigatran): a report of the American College of Cardiology Foundation/ American Heart Association Task Force on practice guidelines. Circulation 2011;123:1144-50.

18. National Collaborating Centre for Chronic Conditions. Atrial Fibrillation: National Clinical Guideline for Management in Primary and Secondary Care. London: Royal College of Physicians 2006.

19. Connolly SJ, Ezekowitz MD, Yusuf S, et al. Dabigatran versus warfarin in patients with atrial fibrillation. N Engl J Med 2009;361:1139-51.

20. Connolly SJ, Ezekowitz MD, Yusuf S, et al. Newly identified events in the RE-LY trial. N Engl J Med 2010;363:1875-6.

21. Fox KA, Piccini JP, Wojdyla D, et al. Prevention of stroke and systemic embolism with rivaroxaban compared with warfarin in patients with non-valvular atrial fibrillation and moderate renal impairment. Eur Heart $J$ 2011;32:2387-94.

22. Muir WW, Bonagura JD. Treatment of cardiac arrhythmias in dogs with gastric distention-volvulus. J Am Vet Med Assoc 1984;184:1366-71.

23. ACTIVE Writing Group of the ACTIVE Investigators, Connolly S, Pogue J, et al. Clopidogrel plus aspirin versus oral anticoagulation for atrial fibrillation in the atrial fibrillation clopidogrel trial with irbesartan for prevention of vascular events (ACTIVE W): a randomized controlled trial. Lancet 2006;367:1903-12.

24. Menke J, Lüthje L, Kastrup A, et al. Thromboembolism in atrial fibrillation. Am $J$ Cardiol 2010;105:502-10. 
This pdf has been created automatically from the final edited text and images.

Copyright 2011 BMJ Publishing Group. All rights reserved. For permission to reuse any of this content visit http://group.bmj.com/group/rights-licensing/permissions.

BMJ Case Report Fellows may re-use this article for personal use and teaching without any further permission.

Please cite this article as follows (you will need to access the article online to obtain the date of publication).

Liu A, Nicol E. Fast atrial fibrillation and caecal volvulus - a case report and evidence based management. BMJ Case Reports 2011;

10.1136/bcr.10.2011.4982, Published XXX

Become a Fellow of BMJ Case Reports today and you can:

- Submit as many cases as you like

- Enjoy fast sympathetic peer review and rapid publication of accepted articles

- Access all the published articles

- Re-use any of the published material for personal use and teaching without further permission

For information on Institutional Fellowships contact consortiasales@bmjgroup.com

Visit casereports.bmj.com for more articles like this and to become a Fellow 